\title{
Exact time evolution of the pair distribution function for an entangled two-electron initial state
}

\author{
I. Nagy, ${ }^{1,2}$ I. Aldazabal, ${ }^{2,3}$ and A. Rubio ${ }^{2,3,4,5}$ \\ ${ }^{1}$ Department of Theoretical Physics, Institute of Physics, Technical University of Budapest, H-1521 Budapest, Hungary \\ ${ }^{2}$ Donostia International Physics Center, P. Manuel de Lardizabal 4, E-20018 San Sebastián, Spain \\ ${ }^{3}$ Centro de Física de Materiales (CSIC-UPVIEHU)-MPC, P. Manuel de Lardizabal 5, E-20018 San Sebastián, Spain \\ ${ }^{4}$ Departamento de Física de Materiales, Facultad de Químicas, Universidad del Pais Vasco, Apartado 1072, E-20018 San Sebastián, Spain \\ ${ }^{5}$ Nano-Bio Spectroscopy Group and ETSF Scientific Development Centre, Avenida de Tolosa 72, E-20018 E-20018 San Sebastián, Spain
}

(Received 1 June 2012; published 20 August 2012)

\begin{abstract}
Based on the correlated ground-state wave function of an exactly solvable interacting one-dimensional twoelectron model Hamiltonian we address the switch-off of confining and interparticle interactions to calculate the exact time-evolving wave function from a prescribed correlated initial state. Using this evolving wave function, the time-dependent pair probability function $\mathcal{R}\left(x_{1}, x_{2}, t\right) \equiv n_{2}\left(x_{1}, x_{2}, t\right) /\left[n\left(x_{1}, t\right) n\left(x_{2}, t\right)\right]$ is determined via the pair density $n_{2}\left(x_{1}, x_{2}, t\right)$ and single-particle density $n(x, t)$. It is found that $\mathcal{R}(0,0, t=\infty)=\mathcal{R}(0,0, t=0)>1$, and $\mathcal{R}\left(x_{1}, x_{2}, t^{*}\right)=1$ at a finite $t^{*}$ for $\Lambda \neq 0$ interparticle interaction strength in the initial two-electron model. By expanding $n(x, t)$ in an infinite sum of closed-shell products of time-dependent normalized single-particle states and time-dependent occupation numbers $P_{k}(\Lambda, t)$, the von Neumann entropy $S(\Lambda, t)=-\sum_{k=0}^{\infty} P_{k}(t) \ln P_{k}(t)$ is calculated as well. The such-defined information entropy is zero at $t^{*}(\Lambda)$ and its maximum in time is $S(\Lambda, t=$ $\infty)=S(\Lambda, t=0)$.
\end{abstract}

DOI: 10.1103/PhysRevA.86.022512

PACS number(s): 31.15.ec, 03.65.-w, 03.67.-a, 71.15.Mb

\section{MOTIVATIONS}

The solution of the time-dependent quantum mechanical many-electron problem is one of the central problems in many important areas of contemporary physics and chemistry. The common interest is mainly driven by experimental and technological progress to probe and control electronic states on the atomic time scale, as, for instance, in attosecond precision time-resolved investigations [1]. The main goal of this study is to investigate physical and easily visualizable quantities like the single-particle probability density $n(x, t)$ and the pair probability density $n_{2}\left(x_{1}, x_{2}, t\right)$, of an interacting two-particle model in the time domain. Their understanding provides [2] transparent and complementary insight into the nature of multiparticle systems. Since the chosen (see below) time-dependent interacting two-electron problem is exactly solvable, the results obtained on such quantities could be useful in alternative approaches like the time-dependent density matrix functional theory [3-6] and the time-dependent density functional theory [7-11] where the basic variables contain less and less information than an exact wave function, but enough to get any physical observable. The quantum information aspect of interacting systems is an important current research topic as well, but only little is known on time-dependent occupation numbers and natural orbitals. Recent numerical works on one-dimensional scattering processes $[4,12]$ herald, for instance, a nonmonotonic change in the associated quantum entropy as a function of time.

This theoretical paper is organized as follows. In Sec. II, we give a detailed analytical derivation of our time-dependent basic quantities. Illustrative figures are added as well. Section III is devoted to a short summary and an outlook.

\section{RESULTS AND DISCUSSION}

According to the above motivation, the present study rests on the exact ground-state solution of a frequently employed
[13-20] two-electron model Hamiltonian

$$
\begin{aligned}
\hat{H}= & -\frac{\hbar^{2}}{2 m}\left(\frac{d^{2}}{d x_{1}^{2}}+\frac{d^{2}}{d x_{2}^{2}}\right)+\frac{1}{2} m \omega_{0}^{2}\left(x_{1}^{2}+x_{2}^{2}\right) \\
& -\frac{1}{2} m \Lambda \omega_{0}^{2}\left(x_{1}-x_{2}\right)^{2}
\end{aligned}
$$

in one space dimension. Using Hartree atomic units (i.e., $\hbar=$ $m=1)$ and the simple canonical transformations $x_{+}=\left(x_{1}+\right.$ $\left.x_{2}\right) / \sqrt{2}$ and $x_{-}=\left(x_{1}-x_{2}\right) / \sqrt{2}$, one gets

$$
\hat{H}=\left[-\frac{1}{2} \frac{d^{2}}{d x_{+}^{2}}+\frac{1}{2} a_{0}^{2} x_{+}^{2}\right]+\left[-\frac{1}{2} \frac{d^{2}}{d x_{-}^{2}}+\frac{1}{2} b_{0}^{2} x_{-}^{2}\right],
$$

where $a_{0}=\omega_{0}$ and $b_{0}=\omega_{0} \sqrt{1-2 \Lambda}$ are convenient shorthands. The repulsive interparticle interaction corresponds to $\Lambda \in[0,0.5]$ and the attractive case to $\Lambda<0$ in our notation. Due to the separability of the Hamiltonian $\hat{H}$, the ground-state wave function $\psi\left(x_{1}, x_{2}\right)$ becomes a product in the above canonical variables

$$
\begin{aligned}
\psi\left(x_{1}, x_{2}\right)= & \phi\left(x_{+}\right) \phi\left(x_{-}\right) \\
= & \left(\frac{a_{0}}{\pi}\right)^{1 / 4} \exp \left(-\frac{1}{2} a_{0} x_{+}^{2}\right)\left(\frac{b_{0}}{\pi}\right)^{1 / 4} \\
& \times \exp \left(-\frac{1}{2} b_{0} x_{-}^{2}\right),
\end{aligned}
$$

but this state is not separable as a simple product in the original coordinates $x_{1}$ and $x_{2}$, when $\Lambda \neq 0$ (i.e., when there is an inseparable entanglement due to electron correlation).

Here we take this product state as an initial state $\Psi\left(x_{1}, x_{2}, t=0\right) \equiv \phi\left(x_{+}\right) \phi\left(x_{-}\right)$and solve the time-dependent Schrödinger equation with a new ( $n$ ) Hamiltonian

$$
\hat{H}_{n}=-\frac{1}{2} \frac{d^{2}}{d x_{+}^{2}}-\frac{1}{2} \frac{d^{2}}{d x_{-}^{2}}
$$

to derive the $\Psi\left(x_{1}, x_{2}, t\right) \equiv \Phi\left(x_{+}, t\right) \Phi\left(x_{-}, t\right)$ function after a sudden quench [10] of all interactions in $\hat{H}$. Practically, due 
to the simplicity of $\hat{H}_{n}$, we can employ the standard

$$
G\left(x_{j}, x_{j}^{\prime}, t\right)=\frac{1}{\sqrt{2 \pi i t}} \exp \left[\frac{i}{2 t}\left(x_{j}-x_{j}^{\prime}\right)^{2}\right]
$$

Green's function (the subscript $j$ refers to + and - ) in a well-known [21] manner

$$
\Phi\left(x_{j}, t\right)=\int_{-\infty}^{\infty} d x_{j}^{\prime} G\left(x_{j}, x_{j}^{\prime}, t\right) \phi\left(x_{j}\right) .
$$

After evaluation of the independently propagating components of $\Psi\left(x_{1}, x_{2}, t\right)$ we get

$$
\begin{aligned}
\Psi\left(x_{1}, x_{2}, t\right)= & \left(\frac{a_{t}}{\pi}\right)^{1 / 4} \exp \left[-\frac{1}{2} a_{t} x_{+}^{2}\left(1-i t a_{0}\right)\right]\left(\frac{b_{t}}{\pi}\right)^{1 / 4} \\
& \times \exp \left[-\frac{1}{2} b_{t} x_{-}^{2}\left(1-i t b_{0}\right)\right]
\end{aligned}
$$

where $a_{t}=a_{0} /\left(1+t^{2} a_{0}^{2}\right)$ and $b_{t}=b_{0} /\left(1+t^{2} b_{0}^{2}\right)$. It is this exact wave function, with a built-in initial correlation, which is used below to generate time-dependent probabilities.

First we derive the normalized one-particle probability density $n(x, t)$ from the diagonal of the reduced one-particle density matrix (one-matrix) in the usual way

$$
n(x, t)=\int_{-\infty}^{\infty} d x_{3} \Psi\left(x_{1}=x, x_{3}, t\right) \Psi^{*}\left(x_{2}=x, x_{3}, t\right) .
$$

After a straightforward calculation we get for this density

$$
n(x, t)=\left(\frac{\Omega_{t}}{\pi}\right)^{1 / 2} e^{-\Omega_{t} x^{2}}
$$

in which $\Omega_{t}=2 a_{t} b_{t} /\left(a_{t}+b_{t}\right)$. Remarkably enough, by using Mehler's formula [22] for generating functions and previous experience $[15,17,19]$, we can decompose this density uniquely (see below) in the following closed-shell-like [23] manner

$$
\begin{aligned}
n(x, t)= & \sum_{k=0}^{\infty}\left[\left(1-\xi_{t}\right)\left(\xi_{t}\right)^{k}\right]\left[\left(\frac{\bar{\omega}_{t}}{\pi}\right)^{1 / 4} \frac{1}{\sqrt{2^{k} k !}}\right. \\
& \left.\times \exp \left(-\frac{1}{2} \bar{\omega}_{t} x^{2}\right) H_{k}\left(\sqrt{\bar{\omega}_{t}} x\right)\right]^{2},
\end{aligned}
$$

in terms of an infinite sum of weighted product of normalized one-particle oscillator states. The underlying constraint behind this mapping is $\Omega_{t} \equiv \bar{\omega}_{t}\left(1-\xi_{t}\right) /\left(1+\xi_{t}\right)$, and it is satisfied with $\xi_{t}=\left(\sqrt{a_{t}}-\sqrt{b_{t}}\right)^{2} /\left(\sqrt{a_{t}}+\sqrt{b_{t}}\right)^{2}$ and $\bar{\omega}_{t}=\sqrt{a_{t} b_{t}}$. At $t \rightarrow 0$ we recover the unique stationary results $(\xi$ and $\bar{\omega}$ ) of earlier works $[15,19]$. We have $\sum_{k=0}^{\infty}\left(1-\xi_{t}\right)\left(\xi_{t}\right)^{k}=1$ for all time, which shows that the evolving probability density $n(x, t)$ is properly normalized.

At this point we stress few remarkable facts. If one is tempted to use instead of Eq. (1) an effective (e), say KohnSham, single-particle Hamiltonian

$$
\hat{H}_{e}=-\frac{1}{2}\left(\frac{d^{2}}{d x_{1}^{2}}+\frac{d^{2}}{d x_{2}^{2}}\right)+\frac{1}{2} \Omega_{e}^{2}\left(x_{1}^{2}+x_{2}^{2}\right),
$$

with a prescribed $\Omega_{e} \equiv 2 a_{0} b_{0} /\left(a_{0}+b_{0}\right)$ to get the precise initial density at $\Lambda \neq 0$, the time-dependent density after the

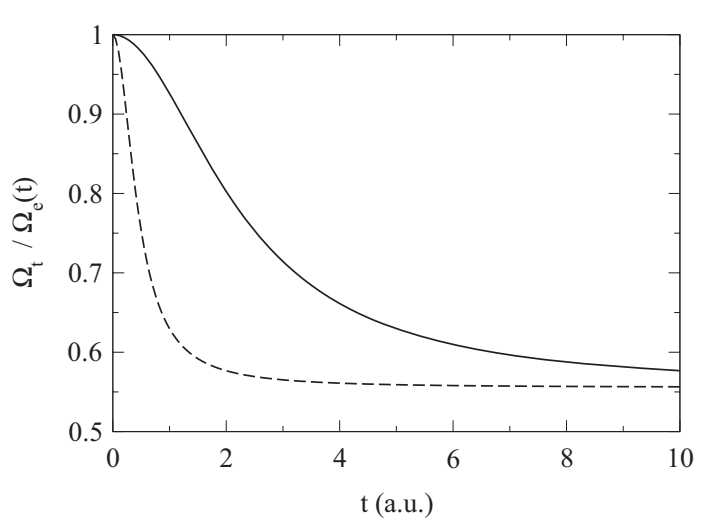

FIG. 1. Calculated dimensionless ratio, defined via $\Omega_{t} / \Omega_{e}(t)$, as a function of time measured in atomic units. The solid curve refers to the repulsive interparticle interaction, while the dashed one to the attractive case. See the text for further details on numerical values used in data generation.

switch-off of the potential in $\hat{H}_{e}$ would be

$$
n_{e}(x, t)=\left[\frac{\Omega_{e}(t)}{\pi}\right]^{1 / 2} e^{-\Omega_{e}(t) x^{2}}
$$

in which $\Omega_{e}(t)=\Omega_{e} /\left(1+t^{2} \Omega_{e}^{2}\right)$ according to the propagation of the auxiliary product form for the initial ground-state wave function. For $t>0$ one has $n_{e}(x, t) \neq n(x, t)$.

Furthermore, one could, from a purely mathematical point of view, reproduce $n(x, t)$ in a point-wise manner by taking, for instance, the $\bar{\omega}_{t} \Rightarrow \Omega_{e}$ replacement in the infinite sum of Eq. (9) together with the $\xi_{t} \Rightarrow \xi(t)=\left[t^{2} a_{0} b_{0} /\left(2+t^{2} a_{0} b_{0}\right)\right]$ change. This is dictated by the above single constraint on two new [here $\Omega_{e}$ and $\xi(t)$ ] variables. Clearly, considering only the decomposition of an exact density $n(x, t)$, one may run into a many-to-one [24] problem. As it is well known, the bijectivity question [25] is crucial in those proofs by which one can, at least in principle, safely apply certain basic variables (say the density) in effective iterative calculations of observable physical quantities in many-body problems. In our case with two electrons, only $\Omega_{t}$ and $\xi_{t}$ provide the unique (see above) decomposition which is continuous in time. We will use the $\xi_{t}$ and $\xi(t)$ representations below, in Eq. (14).

In Fig. 1, we illustrate the $\Omega_{t} / \Omega_{e}(t)$ ratio as a function of time. To generate this ratio we used $\omega_{0}=1$ (a.u.), and the $\Lambda=0.48$ and $\Lambda=-12$ values to model repulsive and attractive interparticle interactions, respectively. These choices for couplings will be applied throughout this study when we exhibit further illustrative figures. They result in a common $\xi_{t}=0.146$ value at $t=0$, independent of the sign of $\Lambda$. We add that this duality behind such a dimensionless quantity holds [17] for any $\Lambda \in[0,0.5]$. The figure shows quite transparently how the output of an effective model can depart from an exact result in time. For the $t \rightarrow \infty$ limit we get $\left[\Omega_{t} / \Omega_{e}(t)\right] \rightarrow\left[2 \sqrt{a_{0} b_{0}} /\left(a_{0}+b_{0}\right)\right]^{2} \leqslant 1$ for any $\Lambda \neq 0$. Thus, we can conclude that the auxiliary single-particle modeling results in a more compact probability distribution for $t>0$ than the exact description.

We turn to the physically important pair density, $n_{2}\left(x_{1}, x_{2}, t\right)$. In our two-electron model it is given by 
$[14,16]$ the probability function $\Psi\left(x_{1}, x_{2}, t\right) \Psi^{*}\left(x_{1}, x_{2}, t\right)$. Thus we get

$$
\begin{aligned}
n_{2}\left(x_{1}, x_{2}, t\right)= & \left(\frac{a_{t} b_{t}}{\pi^{2}}\right)^{1 / 2} \exp \left[-\frac{1}{2}\left(x_{1}^{2}+x_{2}^{2}\right)\left(a_{t}+b_{t}\right)\right] \\
& \times \exp \left[-x_{1} x_{2}\left(a_{t}-b_{t}\right)\right]
\end{aligned}
$$

in the original variables, $x_{1}$ and $x_{2}$, showing the entanglement transparently. Clearly, this function contains more information than the density $n(x, t)$ or the corresponding one matrix. Therefore, statements based on its behavior in time put useful controls on speculations based only on $n(x, t)$. Using $n_{2}\left(x_{1}, x_{2}, t\right)$, we define [14] our central probability distribution function as $\mathcal{R}\left(x_{1}, x_{2}, t\right) \equiv n_{2}\left(x_{1}, x_{2}, t\right) /\left[n\left(x_{1}, t\right) n\left(x_{2}, t\right)\right]$. In the noninteracting case $(\Lambda=0)$, or in an auxiliary single-particle modeling, this pair distribution is unity at any time and $x_{i}$, reflecting the independent motions of particles. Thus, deviations of $\mathcal{R}\left(x_{1}, x_{2}, t\right)$ from unity show the influence of an initial built-in correlation in the time domain. In the long-time $(t \rightarrow \infty)$ limit we have $a_{t} \rightarrow\left(a_{0} t^{2}\right)^{-1}$ and $b_{t} \rightarrow\left(b_{0} t^{2}\right)^{-1}$. Thus, the prefactor in $n_{2}\left(x_{1}, x_{2}\right)$ behaves in this limit as $\sim\left(\bar{\omega} t^{2}\right)^{-1}$, which shows the expected flattening of this physical quantity. Notice that the flattening is weaker in $n(x, t)$ since its prefactor $\propto\left[t \sqrt{a_{0}+b_{0}}\right]^{-1}$ for $t \rightarrow \infty$.

After substitutions and rearrangement we arrive at the following expression

$$
\begin{aligned}
\mathcal{R}\left(x_{1}, x_{2}, t\right)= & \left(\frac{a_{t}+b_{t}}{2 \sqrt{a_{t} b_{t}}}\right) \exp \left[-\frac{1}{2}\left(x_{1}^{2}+x_{2}^{2}\right) \frac{\left(a_{t}-b_{t}\right)^{2}}{a_{t}+b_{t}}\right] \\
& \times \exp \left[-x_{1} x_{2}\left(a_{t}-b_{t}\right)\right] .
\end{aligned}
$$

This distribution function is $\mathcal{R}(0,0, t) \geqslant 1$ at contact. Furthermore, considering the above-stated limits, we get for its prefactor $\mathcal{R}(0,0, t=0)=\mathcal{R}(0,0, t=\infty) \geqslant 1$ for any $\Lambda$. Even more importantly, when $a_{t}=b_{t}$ for $\Lambda \neq 0$ we get a $\mathcal{R}\left(x_{1}, x_{2}, t^{*}\right)=1$ value which appears at $t^{*}=1 / \sqrt{a_{0} b_{0}}=$ $1 / \bar{\omega}$. This $t^{*}$ depends on the sign of $\Lambda$, and for a strong repulsive interparticle interaction, where $\Lambda \in[0,0.5]$, it can be very large since $\bar{\omega} t^{*}=(1-2 \Lambda)^{-1 / 4}$. With an $\bar{\omega}=0.5$ (in a.u.) and a moderate interparticle repulsion $\Lambda=3 / 8$, we are in the typical atomic time scale since $t^{*} \simeq 3$ (i.e., it is about $73 \times 10^{-18} \mathrm{~s}$ in normal units).

In the $\Lambda \rightarrow 0.5$ critical limit (i.e., for strong-enough initial interparticle correlation) this time interval could be even larger. The finite value of time for $\Lambda \neq 0$ signals a time interval (a memory range) within which the pair distribution still resembles for its initial shape in the $x_{1}$ and $x_{2}$ space variables. This similarity is lost for $t>t^{*}$ when there is a sign change in the second exponent of Eqs. (12) and (13). Such a sign-change could occur in the stationary situation only under a sign change of the interaction strength $\Lambda$ in Eq. (1).

In Fig. 2 we plot the $\mathcal{R}(0,0, t)$ probability function as a function of time, which is measured in atomic units. As we prefixed at Fig. 1, we apply $\omega_{0}=1$ and the $\Lambda=$ 0.48 and $\Lambda=-12$ values to model repulsive and attractive interparticle interactions, respectively. The solid curve refers to the repulsion and the dashed curve rests on attraction. With these parameters we get, respectively, about $t^{*} \simeq 2.2$ and $t^{*} \simeq 0.45$. The attractive case needs a shorter time to go through, at its $t^{*} \simeq 0.45$ value, an independent-particle-like situation in which, as we mentioned, $\mathcal{R}\left(x_{1}, x_{2}, t\right)=1$. Figure 2

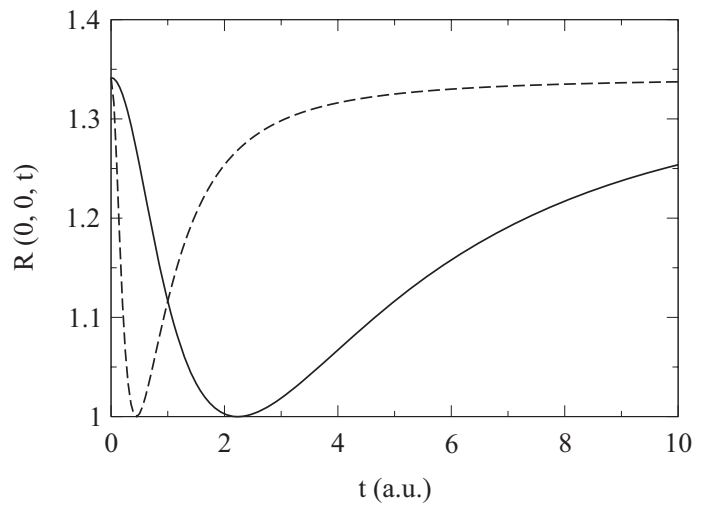

FIG. 2. The dimensionless probability function $\mathcal{R}(0,0, t)$ (i.e., the pair distribution function at the origin $x_{1}=x_{2}=0$, as a function of time $t$ ). The same parameters are used as in Fig. 1. The solid and dashed curves refer to repulsive and attractive interparticle interactions, respectively.

shows that the tendency to the $\mathcal{R}(0,0, t=\infty)=\mathcal{R}(0,0, t=0)$ limit value is slow in the repulsive case. The shape of the dashed curve is more narrow around its $t^{*} \simeq 0.45$. In this (attractive) case the particles were localized more at $x_{1}=x_{2}$ in an attractive external field.

In the light of the above change (at $a_{t}=b_{t}$ for $\Lambda \neq 0$ ) in the time-dependent pair density, we investigate its possible consequence in an entropy determined via our time-dependent natural occupation numbers $P_{k}(\Lambda, t) \equiv\left(1-\xi_{t}\right)\left(\xi_{t}\right)^{k}$ as $S(t)=$ $-\sum_{k=0}^{\infty} P_{k}(t) \ln P_{k}(t)$. Thus, in terms of the unique $\xi_{t}=$ $\left.\left[\left(\sqrt{a_{t}}-\sqrt{b_{t}}\right)^{2} / \sqrt{a_{t}}+\sqrt{b_{t}}\right)^{2}\right] \leqslant 1$ introduced at Eq. (9), we get

$$
S(t)=-\ln \left(1-\xi_{t}\right)-\frac{\xi_{t}}{1-\xi_{t}} \ln \xi_{t},
$$

which is an information-theoretic measure of entanglement on the time scale. The above entropy has zero value, as minimum, at $t^{*}=1 / \sqrt{a_{0} b_{0}}$ for $\Lambda \neq 0$. The maximum depends solely on the interparticle coupling $\Lambda$, and its behavior is $S(t=0)=S(t=\infty)$. Therefore, the information provided by this entropy faithfully reproduce the main characters embodied in the pair distribution function of a two-particle entangled state. If we use, following an earlier [26] work on entanglement dynamics, the so-called linear $(L)$ entropy as an alternative measure we get $S_{L}(t)=2 \xi_{t} /\left(1+\xi_{t}\right)$. This shows the same global behaviors as the von Neumann entropy $S(t)$. If one were tempted to use the auxiliary $\xi(t)$ [see after Eq. (11)] instead of our $\xi_{t}$, the entropies were monotonously growing functions of time.

In Fig. 3 we exhibit the $S(t)$ quantity, an informationtheoretic measure, as a function of time. We can see that not only the mentioned global information (i.e., limiting constant values and sign-dependent $t^{*} \propto(1-2 \Lambda)^{-1 / 4}$ values $)$ are compatible with those exhibited in Fig. 2 for $\mathcal{R}(0,0, t)$, but there is a surprising semiquantitative shape overlap between the corresponding curves over the whole time domain as well. Thus, we may conclude that for our interacting system the investigated quantum-mechanical and information-theoretic measures provide essentially the same details on the time dependence of an initial entanglement. 


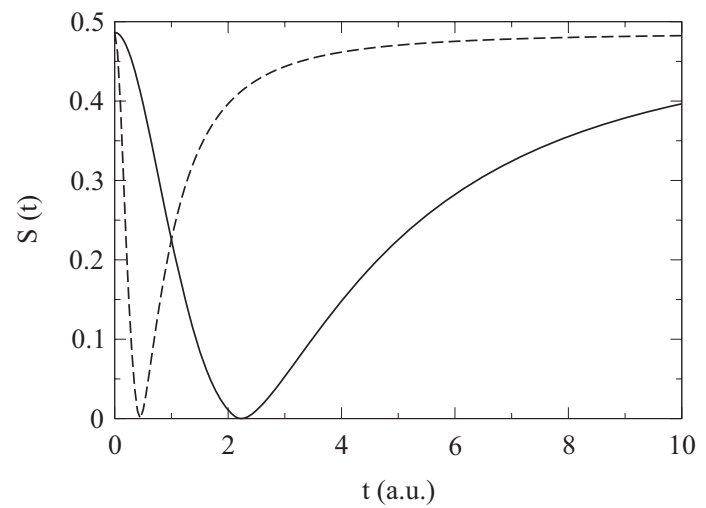

FIG. 3. The information-theoretic entropy $S(t)$, as a function of time $t$ measured in atomic units. The same parameters are used as in Figs. 1 and 2. The solid curve refers to the repulsive interparticle interaction, while the dashed one to the attractive case.

As expected on physical grounds, the time dependence of $S(t)$ found above for our model system is opposite to the dependence found quite recently $[4,12]$ in numerical one-dimensional scattering studies. Now, at this level of understanding trends, we compare our time-dependent information entropy with a time-dependent entropy in thermodynamics. According to a basic book [27] on statistical physics (see Fig. 1 in its paragraph 7), such a dependence could arise in a thermodynamical system for a very large (very improbable) fluctuation at a $t^{*}$. In this case, as Landau concluded after his discussion of the law of entropy increase, one could say that for $t<t^{*}$ and $t>t^{*}$ the system has higher entropy.

We finish this section by a further analogy. Since, as noted first by Srednicki [15], there is a formal mapping at $t=0$ between the above $S(t=0)$ form in Eq. (14) and an ideal thermal oscillator specified by frequency $\bar{\omega}$ and temperature $T=\bar{\omega} / \ln (1 / \xi)$, we are tempted to extend this formal correspondence now to the time domain. We define therefore, using the substitutions of $\bar{\omega} \Rightarrow \bar{\omega}_{t}$ and $\xi \Rightarrow \xi_{t}$ in the above equation for $T$, a new $T_{t}$ function. We get, in such a manner, zero values for $T_{t^{*}}$ at $t^{*}$ due to $\xi_{t^{*}}=0$. Thus, at $t \neq 0$, a zero in our entropy is in harmony with a zero in temperature, in accord with Nernst's theorem [27].

\section{SUMMARY AND OUTLOOK}

Motivated by the theoretical and experimental importance of having a detailed understanding of time-dependent processes on the atomic time scale, an exact solution is given for the time evolvement of a two-electron state under the action of a new Hamiltonian. Specifically, the associated single-particle probability $n(x, t)$, and the pair probability function $n_{2}\left(x_{1}, x_{2}, t\right)$ are determined. The analysis is supplemented by an investigation on the information-theoretic time-dependent entropy $S(t)$.
It is found that the conventional pair probability distribution function $\mathcal{R}\left(x_{1}, x_{2}, t\right)=n_{2}\left(x_{1}, x_{2}, t\right) /\left[n\left(x_{1}, t\right) n_{2}\left(x_{2}, t\right)\right]$ and the von Neumann entropy $S(t)$, both of global nature on entanglement, show consistently a remarkable behavior of the time development of a correlated initial two-electron state. There is a certain $t^{*}$, the magnitude of which depends on the initial correlation, at which the pair distribution function goes through a noninteracting-like state. This time scale is in the atomic range. Within the framework of the applied two-electron model, the analytical results presented here are exact. Thus, we believe that they could provide a useful complement to earlier numerical studies on entropies in time-dependent two-body scattering problems.

As an outlook, we speculate that our theoretical findings could be important in several problems with the initial confinement of interacting (repulsively or attractively) particles, like in ionization problems of atoms [28,29] with transient atomic structures or in electron-hole and electron-electron dynamical processes $[30,31]$ in condensed matter physics. The sign effect of interparticle interaction can result in peculiarities beyond a simple mean-field description applied commonly for charge-neutral [30] pair excitations in metals. Similarly, the interpretation of data obtained in electron-pair emission spectroscopy needs methods beyond an effective one-electron treatment. Indeed, it was emphasized [31] that the very existence of correlation cannot be formulated within a single-particle picture.

Finally, the precise interrelation between time-dependent natural orbitals and laser-dressed Floquet states is a challenging problem to future attempts. Our study may also find applications in the analysis of evolving pairs in optically generated atom-condensates under a tailored change in the sign of interparticle interactions. From a technological perspective, there is an increasing [32] thrust toward engineering ultracold atomic many-body systems for applications in quantum metrology. A deeper understanding of the dynamics in interacting systems is of crucial importance in this context as well.

\section{ACKNOWLEDGMENTS}

I. N. thanks Professor A. Jakovác, Professor N. H. March, and Professor I. V. Tokatly for very useful discussions and acknowledges the warm hospitality at the DIPC. This work has been supported by the Spanish MICINN (Projects No. FIS2010-19609-C02-02 and No. FIS2011-65702-C0201), Grupos Consolidados UPV/EHU del Gobierno Vasco (IT-319-07), Consolider nanoTHERM (Grant No. CSD201000044), ACI-Promociona (ACI2009-1036), European Research Council Advanced Grant DYNamo (ECR-2010-AdGProposal No. 267374), and European Commission projects CRONOS (280879-2 CRONOS CP-FP7) and THEMA (FP7NMP-2008-SMALL-2, 228539).
[1] F. Krausz and M. Ivanov, Rev. Mod. Phys. 81, 163 (2009).

[2] W. Kohn, Rev. Mod. Phys. 71, 1253 (1999).
[3] K. Pernal, O. Gritsenko, and E. J. Baerends, Phys. Rev. A 75, 012506 (2007).

[4] H. Appel and E. K. U. Gross, Europhys. Lett. 92, 23001 (2010). 
[5] R. Requist and O. Pankratov, Phys. Rev. A 83, 052510 (2011).

[6] N. Helbig, J. I. Fuks, I. V. Tokatly, H. Appel, E. K. U. Gross, and A. Rubio, Chem. Phys. 391, 1 (2011).

[7] E. Runge and E. K. U. Gross, Phys. Rev. Lett. 52, 997 (1984).

[8] R. van Leeuwen, Phys. Rev. Lett. 82, 3863 (1999).

[9] I. V. Tokatly, Phys. Rev. B 83, 035127 (2011).

[10] Z.-H. Yang, N. T. Maitra, and K. Burke, Phys. Rev. Lett. 108, 063003 (2012).

[11] P. Elliott and N. T. Maitra, Phys. Rev. A 85, 052510 (2012).

[12] M. G. Benedict, J. Kovács, and A. Czirják, J. Phys. A: Math. Theor. 45, 085304 (2012).

[13] M. Moshinsky, Am. J. Phys. 36, 52 (1968).

[14] E. R. Davidson, Reduced Density Matrices in Quantum Chemistry (Academic, New York, 1976), pp. 14-19.

[15] M. Srednicki, Phys. Rev. Lett. 71, 666 (1993).

[16] N. H. March, I. A. Howard, I. Nagy, and A. Rubio, Phys. Lett. A 288, 101 (2001).

[17] J. Pipek and I. Nagy, Phys. Rev. A 79, 052501 (2009).

[18] H. G. Laguna and R. P. Sagar, Phys. Rev. A 84, 012502 (2011).

[19] I. Nagy and I. Aldazabal, Phys. Rev. A 84, 032516 (2011); 85, 034501 (2012).

[20] Ph. Blanchard, J. Gracia-Bondía, and J. C. Várilly, Int. J. Quantum Chem. 112, 1134 (2012).
[21] D. ter Haar, Problems in Quantum Mechanics (Pion, London, 1975).

[22] A. Erdélyi, Higher Transcendental Functions (McGraw-Hill, New York, 1953), p. 194.

[23] P.-O. Löwdin and H. Shull, Phys. Rev. 101, 1730 (1956).

[24] V. Sahni and X.-Y. Pan, Phys. Rev. A 85, 052502 (2012).

[25] R. M. Dreizler and E. K. U. Gross, Density Functional Theory (Springer-Verlag, Berlin, 1990).

[26] F. Buscemi, P. Bordone, and A. Bertoni, Phys. Rev. A 75, 032301 (2007).

[27] L. D. Landau and E. M. Lifshitz, Statistical Physics (Butterworth-Heinemann, Oxford, 1980).

[28] R. Pazourek, J. Feist, S. Nagele, and J. Burgdörfer, Phys. Rev. Lett. 108, 163001 (2012).

[29] N. Shivaram, H. Timmers, X.-M. Tong, and A. Sandhu, Phys. Rev. Lett. 108, 193002 (2012).

[30] R. Díez Muiño, D. Sánches-Portal, V. M. Silkin, E. V. Chulkov, and P. M. Echenique, Proc. Natl. Acad. Sci. USA 108, 971 (2011), and references therein.

[31] F. O. Schumann, R. S. Dhaka, G. A. van Riessen, Z. Wei, and J. Kirschner, Phys. Rev. B 84, 125106 (2011), and references therein.

[32] A. Polkovnikov, K. Sengupta, A. Silva, and M. Vengalattore, Rev. Mod. Phys. 83, 863 (2011). 